\title{
Utilisation of early intervention services in young children with hypoplastic left heart syndrome
}

Kathleen A. Mussatto, ${ }^{1}$ Danielle Hollenbeck-Pringle, ${ }^{2}$ Felicia Trachtenberg, ${ }^{2}$ Erica Sood, ${ }^{3}$ Renee Sananes, ${ }^{4}$ Nancy A. Pike, ${ }^{5}$ Linda M. Lambert, ${ }^{6}$ William T. Mahle, ${ }^{7}$ David J. Goldberg, ${ }^{8}$ Caren S. Goldberg, ${ }^{9}$ Carolyn Dunbar-Masterson, ${ }^{10}$ Michelle Otto, ${ }^{11}$ Bradley S. Marino, ${ }^{12}$ Bronwyn H. Bartle, ${ }^{13}$ Ismee A. Williams, ${ }^{14}$ Jeffrey P. Jacobs, ${ }^{15}$ Sinai C. Zyblewski, ${ }^{16}$ Victoria L. Pemberton ${ }^{17}$

${ }^{1}$ Children's Hospital of Wisconsin, Herma Heart Center, Milwankee, Wisconsin; ${ }^{2}$ New England Research Institutes, Inc., Watertown, Massachusetts; ${ }^{3}$ Nemours/Alfred I. duPont Hospital for Children, Department of Pediatrics, Wilmington, Delaware, United States of America; ${ }^{4}$ The Hospital for Sick Children, Department of Pediatrics, Toronto, Ontario, Canada; ${ }^{5}$ Children's Hospital Los Angeles, Department of Pediatrics, Los Angeles, California; 'Primary Children's Hospital, Department of Pediatrics, Salt Lake City, Utab; 'Children's Healthcare of Atlanta, Department of Pediatrics, Atlanta, Georgia; ${ }^{8}$ Children's Hospital of Philadelphia, Department of Pediatrics, Pbiladelphia, Pennsylvania; ${ }^{9}$ University of Michigan Health System, Department of Pediatrics, Ann Arbor, Michigan; ${ }^{10}$ Boston Children's Hospital, Department of Pediatrics, Boston, Massachusetts; ${ }^{11}$ Medical College of Wisconsin, Department of Pediatrics, Milwaukee, Wisconsin; ${ }^{12}$ Ann E Robert H. Lurie Children's Hospital, Department of Pediatrics, Chicago, Illinois; ${ }^{13}$ Duke University Hospital, Department of Pediatrics, Durbam, North Carolina; ${ }^{14}$ Department of Pediatrics, New York State Psychiatric Institute, Department of Developmental Neuroscience, Morgan Stanley Children's Hospital of New York-Presbyterian, New York, New York; ${ }^{15}$ Johns Hopkins All Children's Hospital, Johns Hopkins University, Department of Pediatrics, Saint Petersburg, Florida and Baltimore, Pediatric Heart Network, Maryland; ${ }^{16}$ Medical University of South Carolina, Department of Pediatrics, Charleston, South Carolina; ${ }^{17}$ National Heart, Lung, and Blood Institute, Bethesda, Pediatric Heart Network, Maryland, United States of America

Abstract Objective: Children with hypoplastic left heart syndrome are at a risk for neurodevelopmental delays. Current guidelines recommend systematic evaluation and management of neurodevelopmental outcomes with referral for early intervention services. The Single Ventricle Reconstruction Trial represents the largest cohort of children with hypoplastic left heart syndrome ever assembled. Data on life events and resource utilisation have been collected annually. We sought to determine the type and prevalence of early intervention services used from age 1 to 4 years and factors associated with utilisation of services. Methods: Data from 14-month neurodevelopmental assessment and annual medical history forms were used. We assessed the impact of social risk and geographic differences. Fisher exact tests and logistic regression were used to evaluate associations. Results: Annual medical history forms were available for 302 of 314 children. Greater than half of the children (52-69\%) were not receiving services at any age assessed, whereas 20-32\% were receiving two or more therapies each year. Utilisation was significantly lower in year $4(31 \%)$ compared with years $1-3$ (with a range from 40 to $48 \%)(\mathrm{p}<0.001)$. Social risk factors were not associated with the use of services at any age but there were significant geographic differences. Significant delay was reported by parents in $18-43 \%$ of children at ages 3 and 4. Conclusion: Despite significant neurodevelopmental delays, early intervention service utilisation was low in this cohort. As survival has improved for children with hypoplastic left heart syndrome, attention must shift to strategies to optimise developmental outcomes, including enrolment in early intervention when merited.

Keywords: CHD; neurodevelopmental disorders; early intervention

Received: 1 May 2017; Accepted: 13 July 2017; First published online: 29 August 2017

Correspondence to: K. A. Mussatto, Children's Hospital of Wisconsin, 9000 Wisconsin Avenue, Milwaukee, WI 53201, United States of America. Tel: 414266 2073; Fax: 414266 6248; E-mail: kmussatto@chw.org 


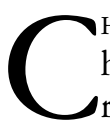
HILDREN WITH COMPLEX CHD, SUCH AS hypoplastic left heart syndrome, are at a high risk for developmental delays. ${ }^{1-6}$ Survival of these patients is increasing. ${ }^{7}$ Neurodevelopmental and psychosocial issues are common long-term morbidities faced by this population. In the Pediatric Heart Network's Single Ventricle Reconstruction Trial, $44 \%$ of children who returned for neurodevelopmental assessment with the Bayley Scales of Infant Development-II at 14 months of age had psychomotor development index scores greater than 2 standard deviations below the population mean $(<70){ }^{8}$ Parent reports of development at 3 years of age for children in the Single Ventricle Reconstruction Trial were significantly lower than average in all domains of the Ages and Stages Questionnaire that assesses cognitive, problem-solving, personal/social, and gross and fine motor skills. ${ }^{9}$ Multiple studies have described a characteristic pattern of mild or combined disabilities in the areas of visual motor integration, language, motor skills, attention, executive function, and behaviour in children with complex heart disease. ${ }^{10,11}$

In the United States of America, all children with complex CHD believed to be at a high risk for developmental delay are eligible for evaluation using early intervention programmes that are mandated by Part $\mathrm{C}$ of the Individuals with Disabilities Education Act. ${ }^{12}$ Early intervention is a broad term that includes individualised, systematic evaluation followed by interventions designed to address atneed areas such as general developmental support, physical, occupational, and/or speech therapy. ${ }^{13}$ Administration of early intervention and interpretation of Individuals with Disabilities Education Act Part C vary widely by state. ${ }^{14,15}$ Typically, therapeutic efforts to support development are offered to children aged $0-3$ years. If support is still needed after age 3 years, children enter early education services with a focus on school readiness.

Despite the high incidence of developmental delay, relatively little is known about the utilisation of early intervention services among children with hypoplastic left heart syndrome. The Single Ventricle Reconstruction trial cohort, enrolled in infancy and followed up through early childhood in the Single Ventricle Reconstruction Extension Study, provided a unique opportunity to investigate the use of early intervention services over time in a geographically diverse sample. Our specific aims were to determine the type and prevalence of early intervention services used from age 1 to 4 years in trial participants, to assess the association between social risk factors and early intervention utilisation, and to investigate whether there were geographic differences in early intervention utilisation.

\section{Materials and methods}

The Single Ventricle Reconstruction Trial has been previously described. ${ }^{7}$ The primary trial endpoint was transplant-free survival at 12 months of age. A secondary endpoint was neurodevelopmental outcomes at 14 months of age as assessed using the Bayley Scales of Infant Development-II. ${ }^{8}$ Capitalising on the largest prospective cohort of children with hypoplastic left heart syndrome who survived initial palliation and remained free from transplant, the Single Ventricle Reconstruction Extension study is a longitudinal follow-up study that maintains annual contact with the study participants through

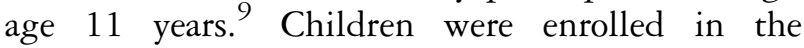
Single Ventricle Reconstruction Extension study at their 14-month Single Ventricle Reconstruction Trial visit. Information on survival status, child development, and use of early intervention services including speech or language therapy, physical therapy, occupational therapy, and treatment for cognitive, psychological, or behavioural disorders was collected annually from parents of study participants.

Parental permission was obtained to maintain annual follow-up and to use data from the 14-month Single Ventricle Reconstruction trial visit and Single Ventricle Reconstruction Extension visits. All Single Ventricle Reconstruction Extension study participants with completed Annual Medical History forms were eligible for this study. Answers to questions, such as which therapies were received, not reported by parents were considered missing for that year. No children were excluded based on medical status, gender, race, or ethnicity. Parents completed the Ages and Stages Questionnaire when the child reached ages 3 and 4 years. This provided subjective assessment of the child's development in five domains: communication, gross motor, fine motor, problem-solving, and personal/social. .,16 $^{9}$

Social risk was assessed using a composite measure of seven aspects of social status adapted from previous work: ${ }^{17-20}$

1. Race/ethnicity of child: white, non-Hispanic versus non-white, Hispanic, or other race

2. Maternal age at child's birth $-\geqslant 21$ years versus $<21$ years - only available from children who participated in a genetic sub-study to the Single Ventricle Reconstruction Trial

3. Maternal education: high-school graduate versus $<$ high-school graduate

4. Child's living arrangement: two adults versus one adult

5. Insurance type: any private versus none or public

6. Language used at home: English versus nonEnglish 
7. Neighbourhood poverty index: percentage of residents below US poverty line $(0-10 \%$ versus $>10 \%)$

Social risk was determined from data provided at the 14-month Single Ventricle Reconstruction Trial visit. Scores for all criteria were summed with equal weights to create a cumulative social risk score (with a range from 0 to 7 ). For each variable a score of 0 was assigned to the lower-risk category and a score of 1 to the higher-risk category, for a maximum possible score of 7. If 1-2 social risk factors were missing, the low-risk score (0) was assigned and a score was calculated. If more than two social risk factors were missing the score was not calculated. The social risk score was treated as a continuous variable. The individual risk factors were also analysed as binary predictors of utilisation of early intervention services.

Geographic differences were assessed by mapping the address of each child at the time of Single Ventricle Reconstruction Trial randomisation into one of four United States Census Regions Northeast, Midwest, South, and West - or to outside of the United States of America. Receipt of any early intervention service by region was determined.

\section{Statistics}

Descriptive statistics were used to illustrate the rates and types of early intervention utilisation. Social risk factors are presented as count and percentage. The rate of early intervention services at each age was determined. Rates, frequency, and duration were calculated for any early intervention and for each specific therapy: speech, physical, occupational, cognitive, and psychological or behavioural. Fisher exact tests were used to determine the association between receipt of therapy in the first year of life and low Bayley Scales of Infant Development-II scores $(<70)$ at 14 months of age.

Logistic regression analyses were performed with receipt of any early intervention as the dependent variable and social risk as an independent variable, after controlling for developmental status - delayed versus not delayed - obtained using the Bayley Scales of Infant Development-II or the Ages and Stages Questionnaire. The relationship between 14-month Bayley Scales of Infant Development-II scores and 3-year Ages and Stages Questionnaire scores was not linear. Locally weighted scatterplot smoothing curves were used to estimate the relationship of Bayley Scales of Infant Development-II scales with the Ages and Stages Questionnaire domains, details of which are available at www.jpeds.com. ${ }^{8}$ For this study, delay was defined as scores $>2$ standard deviations below the mean on both instruments $(<70)$. Odds ratios and $95 \%$ confidence intervals were calculated.
A simple logistic regression was used to explore the relationship between social risk scores and Bayley scores. To evaluate geographic differences in early intervention utilisation, Fisher exact tests were used to assess differences in early intervention utilisation by United States Census Region for receipt of any therapy and for the individual therapies across all 4 years.

All analyses were conducted using Statistical Analysis Software v9.3 (SAS Institute Inc., Cary, North Carolina, United States of America), and statistical significance was tested at level $\mathrm{p}<0.05$.

\section{Results}

There were 314 children in the Single Ventricle Reconstruction Extension study who were alive and transplant-free at age 4. Annual follow-up reports from parents of children ages $1-4$ years were available for 302 children. The prevalence and type of early intervention services used by year are shown in Table 1. Complete data on early intervention utilisation were available for 288-296 children each year. Greater than half of these children were not receiving early intervention services at any of the ages assessed, whereas $20-32 \%$ of the children were receiving two or more therapies in each year. The most common combination of therapies was speech, physical, and occupational therapy. Physical therapy was most common in year 1 and 2, whereas speech therapy was most common in years 3 and 4 . There was a significant difference in the average number of therapies across years $(\mathrm{p}<0.001)$, with the largest difference at year 4 compared with years $1-3$. In year $4,31 \%$ of children received at least one therapy compared with $48 \%$ in year 1 .

Among participants who completed the 14-month visit and all 3 follow-up surveys at years 2,3 , and 4 $(n=274), 38 \%$ reported no early intervention, $20 \%$ reported early intervention services at all 4 time points, $12 \%$ reported early intervention services in the first 2 years only, $8 \%$ in the first 3 years only, and $4 \%$ used early intervention only in their first year of life. Only $8 \%$ reported initiating early intervention services after the first year of life. An additional 10\% reported varying years of early intervention service utilisation.

In order to understand whether early developmental delay resulted in appropriate referrals, the differences in early intervention utilisation for children with delays at 14 months compared with those without delay were examined. At age 14 months, $43 \%$ of the sample had psychomotor development index scores $>2$ standard deviations below the mean $(<70) ; 15 \%$ had mental development index scores $>2$ standard deviations below the mean $(<70)$. Children with significant delays noted during Bayley Scales of 
Table 1. Prevalence and type of early intervention services used by Single Ventricle Reconstruction Extension Trial participants.

\begin{tabular}{lcrrr}
\hline & Age 1 & Age 2 & Age 3 & Age 4 \\
\hline Number of therapies received & & & & \\
0 & $149(52 \%)$ & $154(53 \%)$ & $177(60 \%)$ & $204(69 \%)$ \\
1 & $56(19 \%)$ & $41(14 \%)$ & $41(14 \%)$ & $34(11 \%)$ \\
2 & $38(13 \%)$ & $38(13 \%)$ & $30(10 \%)$ & $27(9.1 \%)$ \\
3 & $39(13 \%)$ & $47(16 \%)$ & $39(13 \%)$ & $28(9.5 \%)$ \\
4 & $7(2 \%)$ & $8(3 \%)$ & $7(2 \%)$ & $3(1 \%)$ \\
Therapy & & & & $70(23 \%)$ \\
Speech/language & $73(24 \%)$ & $81(27 \%)$ & $76(28 \%)$ & $58(19 \%)$ \\
Physical therapy (PT) & $103(34 \%)$ & $100(33 \%)$ & $62(21 \%)$ & $50(17 \%)$ \\
Occupational therapy (OT) & $72(24 \%)$ & $81(27 \%)$ & $24(8 \%)$ & $6(2 \%)$ \\
Cognitive disorder intervention & $29(10 \%)$ & $28(9 \%)$ & $8(3 \%)$ & $6(2 \%)$ \\
Unknown & $13(4 \%)$ & $14(5 \%)$ & $34(12 \%)$ & $27(9 \%)$ \\
Most common combinations & & & $6(2 \%)$ \\
Speech + PT +OT & $33(11 \%)$ & $38(13 \%)$ & $7(2 \%)$ & $12(4 \%)$ \\
PT only & $29(10 \%)$ & $17(6 \%)$ & $22(8 \%)$ & $25(8 \%)$ \\
PT +OT & $16(6 \%)$ & $15(5 \%)$ & & \\
Speech only & $10(4 \%)$ & $12(4 \%)$ & & \\
\hline
\end{tabular}

Infant Development-II assessment - psychomotor development index or mental development index $<70-$ at 14 months of age were significantly more likely to be receiving early intervention services in the first 3 years of life $(\mathrm{p}<0.01)$. This indicates that appropriate referrals were made based on early assessments for many children; however, not all children with delays in the 14-month Bayley Scales of Infant Development-II were receiving early intervention services. For those with a psychomotor development index $<70(n=116)$, only $68 \%$ were receiving early intervention at year 1 . This decreased to $52 \%$ by year 4 . The use of early intervention services for children with psychomotor development index scores $<70$ versus $>70$ at 14 months is illustrated in Figure 1. For those with a mental development index score $<70(n=40), 75 \%$ were receiving early intervention at year 1 . This decreased to $65 \%$ by year 4 . Developmental delays persisted into years 3 and 4 as demonstrated by parent reports for the Ages and Stages Questionnaire. Depending on the specific domain assessed, significant delays were identified in 18-43\% of the children in the following areas: communication, 19-20\%; gross motor, 30\%; fine motor, 35-43\%; problem-solving, 20-23\%; and personal/social, 18-19\%. There was no significant change in Ages and Stages Questionnaire scores from year 3 to year 4 . The prevalence of significant delay at each year is illustrated by the summary statistics for the Bayley Scales of Infant Development-II and the Ages and Stages Questionnaire as shown in Table 2.

Of the 302 participants, 21 could not be assigned a social risk score because of missing data. Among the rest, 106/281 (38\%) had a social risk score of 0 as shown in Figure 2. Non-white race or Hispanic ethnicity

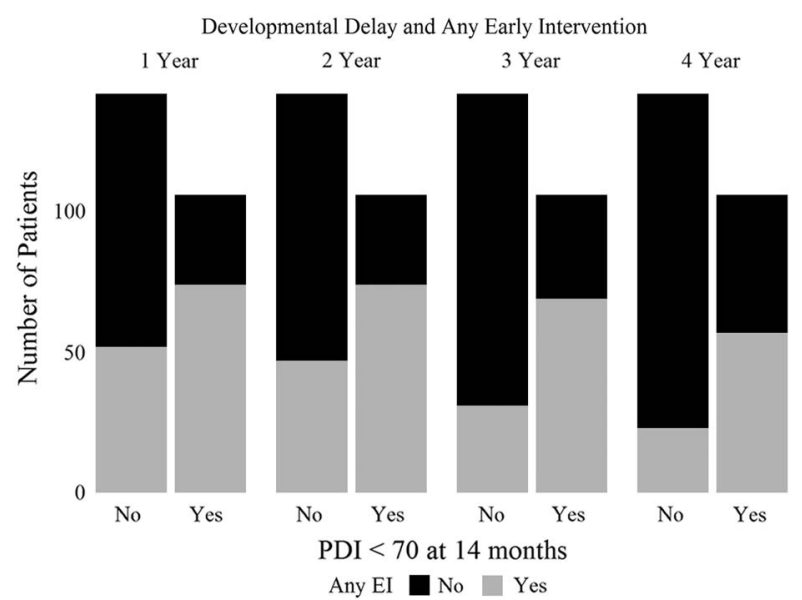

Figure 1.

Early developmental delay and utilisation of early intervention. PDI = Psychomotor Development Index.

was reported by $33 \%$ of the sample. Of the mothers, $6 \%$ were less than 21 years old at the time of their infant's birth. Of note, maternal age was only obtained from children participating in a genetic sub-study of the Single Ventricle Reconstruction Trial, thus available for only 192/302 (64\%) of the mothers. In all, $10 \%$ of the mothers had less than a high-school education. In $18 \%$ the language used at home was non-English or unknown. Overall, $66 \%$ of the children lived in neighbourhoods where the percentage of residents below the United States poverty line was $>10 \%$. Of the children, $8 \%$ lived in a home with a single adult. A total of $37 \%$ of the children had no or only public health insurance. A higher social risk score was significantly associated with an increased likelihood of having a mental development index score $<70$ during the 14-month Bayley Scales of 
Table 2. Summary statistics for Bayley Scales of Infant Development Version II (BSID-II) and Ages and Stages Questionnaire (ASQ)**

\begin{tabular}{lc}
\hline Neurodevelopment measurement & $\mathbf{n}(\%)$ \\
\hline 14 month BSID-II & \\
PDI score <70 & $116 / 273(42 \%)$ \\
MDI score <70 & $40 / 274(15 \%)$ \\
Year 3 ASQ (>2 SD below normal mean) & 198 \\
Communication & $40(20 \%)$ \\
Gross motor & $60(30 \%)$ \\
Fine motor & $70(35 \%)$ \\
Problem-solving & $47(24 \%)$ \\
Personal/social & $35(18 \%)$ \\
Year 4 ASQ (>2 SD below normal mean) & 234 \\
Communication & $45(19 \%)$ \\
Gross motor & $72(31 \%)$ \\
Fine motor & $100(43 \%)$ \\
Problem-solving & $47(20 \%)$ \\
Personal/social & $46(20 \%)$ \\
\hline
\end{tabular}

MDI $=$ Mental Development Index; PDI = Psychomotor Development Index; $\mathrm{SD}=$ standard deviation

*These results have been previously published ${ }^{7,8}$

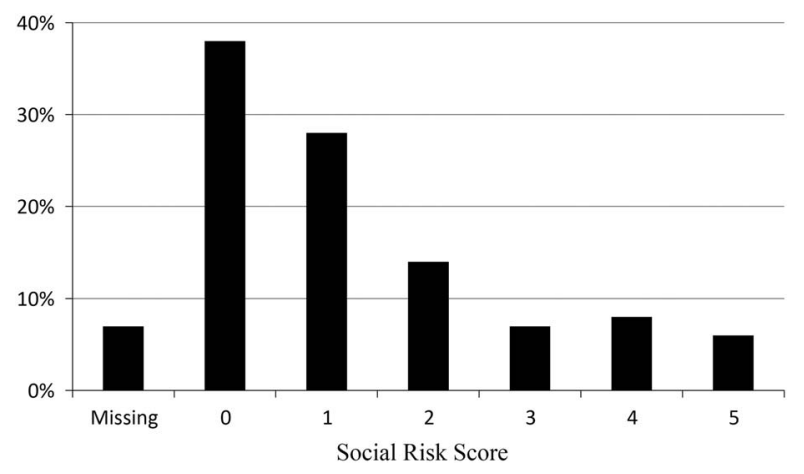

Figure 2 .

Distribution of social risk scores.

Infant Development-II assessment (OR 1.28, 95\% CI $1.05,1.57, \mathrm{p}<0.02)$. Neither the individual social risk factors nor the cumulative social risk score was associated with receipt of early intervention services at any age.

There were significant geographic differences in the utilisation of early intervention services across the four United States census regions. The highest use of any early intervention across all years occurred in the Northeast $-74 \%$ of children - and the lowest occurrence was in the West $-50 \%$ of children - as demonstrated using Fisher's exact test, $\mathrm{p}=0.03$ (Fig $3)$. Of the nine children who were from outside of the United States of America, six (67\%) received some early intervention services.

\section{Discussion}

This cohort has contributed a considerable amount of information about the natural history following surgical palliation of hypoplastic left heart syndrome and resource utilisation in this complex population. It was surprising to discover that less than half of the Single Ventricle Reconstruction Extension study participants were receiving early intervention services at any given year during their first 4 years of life despite the complex nature of their medical conditions. Overall, $38 \%$ of the children never received any early intervention services from ages $1-4$ years. The prevalence of significant delays reported by parents on the Ages and Stages Questionnaire at ages 3 and 4 was high. Scores $>2$ standard deviations below the population mean were reported in $18-43 \%$ of children. This is $7-17$ times higher than the expected prevalence of $2.5 \%$ in a healthy population. ${ }^{21}$ Despite the known risk, developmental delays in children with hypoplastic left heart syndrome may be overlooked.

Practice has changed since the Single Ventricle Reconstruction trial cohort was established for the birth years 2005-2008. The American Heart Association/American Academy of Pediatrics guideline published in $2012^{22}$ now recommends that children with hypoplastic left heart syndrome be evaluated and referred for early intervention services throughout childhood to assess not only cognitive development but also behavioural, psychosocial and adaptive functioning; however, the majority of the cohort had already completed their 4-year Ages and Stages Questionnaire evaluations before this guideline was published. Nevertheless, a great deal of research existed identifying that children with hypoplastic left heart syndrome were at a high risk for delay. Evaluation of developmental outcomes was a secondary aim of the Single Ventricle Reconstruction trial at its inception.

Today, numerous cardiac neurodevelopmental follow-up programmes have been incorporated into comprehensive congenital heart centres. ${ }^{23,24}$ Evaluation and referral for early intervention services should occur as early as possible and are often the standard of practice at hospital discharge for infants with CHD. The importance of repeated evaluation has been illustrated in numerous studies as exposure to risk factors and the prevalence of delay change over time. $^{6,11,25,26}$ In this cohort, motor delays were evident in $43 \%$ of the sample at 14 months of age. These deficits persisted, with both fine and gross motor delays at 3 and 4 years of age in $30-43 \%$ of the sample. Despite these findings, only $19-34 \%$ of the children were receiving physical therapy. As another example, speech therapy in years 1-2 is most likely related to feeding issues, whereas in years 3-4 it is likely related to language skills. Early feeding difficulties have been shown to be important predictors of later developmental delays. ${ }^{6,25,27,28}$ Infants who 


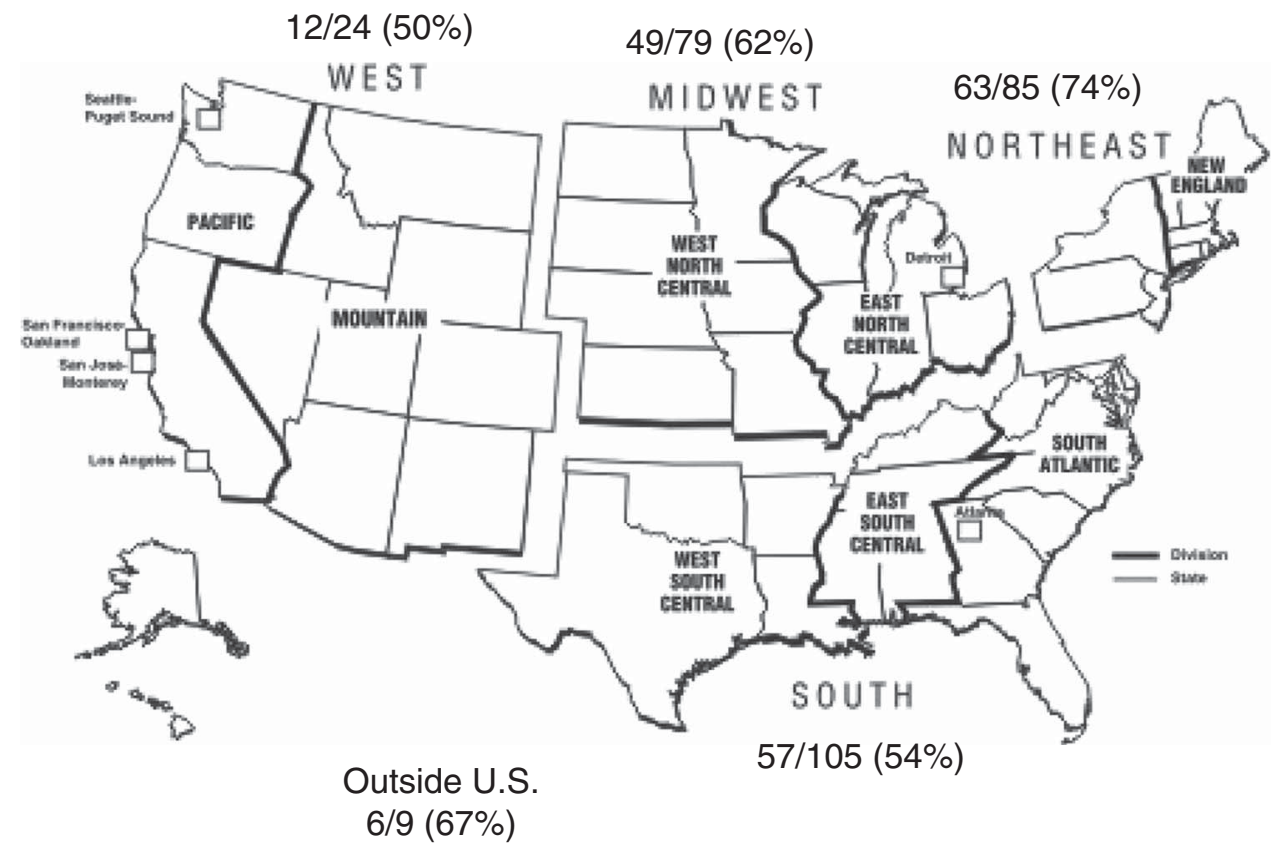

Figure 3.

Utilisation of early intervention services by United States census region, n (\%).

require early speech therapy may warrant increased vigilance to monitor for further delays.

An expert panel of child development specialists has recommended that early intervention be started within 2 months of diagnosis in preterm infants in order to capitalise on the neuroplasticity of the infant brain, ${ }^{29}$ which may allow early intervention to be more effective. ${ }^{30}$ Infants with CHD have been shown to have delayed brain maturation, ${ }^{31}$ much like preterm infants. This emphasises the importance of engaging in early intervention to prevent or reduce long-term problems. The most common finding in young children with CHD has been a high prevalence of low-severity developmental delays. ${ }^{6}$ These delays may go undetected without formal evaluation. Further, mild delay early in life may progress to more significant disability as developmental tasks become more complex, and mild delay in one area may compromise development in other areas. Mild delays may also be more amenable to remediation early in life. ${ }^{17}$

Use of early intervention services decreased over time from a high of $48 \%$ at year 1 to a low of $31 \%$ at year 4; yet, there was no corresponding decrease in the prevalence of developmental delay. In all, $20 \%$ of the children were receiving some form of early intervention services during all 4 years. Only $8 \%$ of children entered therapy at some point after 1 year of age. It appears that if children do not receive early intervention services soon, they may never receive them. Receipt of early intervention services in year 4 was significantly lower than in years $1-3$. This is important because most states offer coordinated birth-to- 3 services and early intervention after age 3 years requires another referral for preschool services. This may present an obstacle for families whose children would still benefit from support.

Studies have shown that the use of early intervention services can be influenced by many factors. There are state and regional variations in access to services and eligibility criteria. ${ }^{15}$ Children with mild delays are less likely to receive services. ${ }^{17}$ Children who do not have recognisable conditions, such as major genetic syndromes or visible birth defects, may be less likely to receive early intervention. ${ }^{18}$ Social risk factors, including both family and neighbourhood factors, have also been shown to influence child development and use of early intervention services. ${ }^{17-20}$

Contrary to previous research ${ }^{17-20}$ we did not find that social risk factors had a negative influence on utilisation of early intervention services; however, social risk was associated with a greater prevalence of developmental delay. A greater percentage of people living below the poverty level within a child's census block has been previously shown to be an independent predictor of abnormal domains on the Ages and Stages Questionnaire at 3 years of age in this cohort. ${ }^{9}$ Despite social risk factors, it may be that parents or clinicians caring for these children were particularly motivated to seek early intervention because of known risk. Participation in the Single Ventricle Reconstruction Trial may also have educated parents about the need for ongoing evaluation and 
developmental support. If a study participant was found to be outside the normal range for one or more areas on the Ages and Stages Questionnaire, the parent(s) and primary care provider were notified and encouraged to seek appropriate intervention. We did identify regional differences in use of early intervention services across the United States of America. Although early intervention services are mandated by Part $\mathrm{C}$ of the Individuals with Disabilities Education Act in the United States of America, there is wide variability in interpretation and eligibility by state. $^{14,17}$

There are important limitations to this study, the most significant of which is that we relied on parent reports of use of early intervention services. Parents may not have distinguished between an evaluation and actual therapy. We also were unable to quantify the dose or the quality of the early intervention services received. Further, we did not know what recommendations parents received from their primary care providers. We did not collect data on why intervention services were not received. Possible explanations include lack of referral, child condition, parent preference, lack of family resources or lack of access to early intervention programmes. There were missing data for some variables. Most notably, maternal age at infant birth was only available for $64 \%$ of the mothers and this could potentially introduce bias into the social risk score. Social risk was assessed based on demographic information at a single time point in the Single Ventricle Reconstruction Trial. Data were not available to account for changes in demographics over time such as advancements in education, relocation, or changes in insurance coverage.

In conclusion, less than half of the children received early intervention services in any given year during their first 4 years of life. The prevalence of developmental delay was markedly higher than what is expected in a healthy population. Current guidelines recommend all children with complex CHD undergo repeated developmental evaluation and referral for early intervention throughout childhood. As survival has become the expectation for the majority of children with hypoplastic left heart syndrome, it is our obligation to ensure everything is done to help them achieve their full potential.

\section{Acknowledgements}

The authors would like to thank the administrative support staff of New England Research Institute - in particular, Tanya Olesker and Beverly Slayton - for facilitating this project. The authors thank the centres participating in the Single Ventricle Reconstruction Extension study for follow-up data collection.
The authors also thank the children and parents for their participation.

\section{Financial Support}

Supported by grants from the National Heart, Lung, and Blood Institute: HL068269, HL068270, HL068279, HL068281, HL068285, HL068292, HL068290, HL068288, HL085057, HL109781, and HL109737. This work is solely the responsibility of the authors and does not necessarily represent the official views of NHLBI or NIH.

\section{Conflicts of Interest}

None.

\section{Ethical Standards}

The authors assert that all procedures contributing to this work comply with the ethical standards of the relevant national guidelines on human experimentation Department of Health and Human Services Code of Federal Regulations (45 CFR 46) Policy for Protection of Human Research Subjects and with the Helsinki Declaration of 1975, as revised in 2008, and has been approved by the respective institutional review boards or research ethics boards of the Children's Hospital of Wisconsin, the New England Research Institutes, Nemours/Alfred I. duPont Hospital for Children, The Hospital for Sick Children, Toronto, Children's Hospital Los Angeles, Primary Children's Hospital, Children's Healthcare of Atlanta, Children's Hospital of Philadelphia, University of Michigan Health System, Boston Children's Hospital, Medical College of Wisconsin, North Carolina Consortium, Morgan Stanley Children's Hospital of New YorkPresbyterian, Johns Hopkins All Children's Hospital, Medical University of South Carolina, and Cincinnati Children's Hospital and Medical Center.

\section{References}

1. Wernovsky G. Current insights regarding neurological and developmental abnormalities in children and young adults with complex congenital cardiac disease. Cardiol Young. 2006; 16 (Suppl 1): 92-104.

2. Majnemer A, Limperopoulos C, Shevell MI, Rohlicek C, Rosenblatt B, Tchervenkov C. A new look at outcomes of infants with congenital heart disease. Pediatr Neurol. 2009; 40: 197-204.

3. Snookes SH, Gunn JK, Eldridge BJ, et al. A systematic review of motor and cognitive outcomes after early surgery for congenital heart disease. Pediatrics 2010; 125: e818-e827.

4. Gaynor JW, Gerdes M, Nord AS, et al. Is cardiac diagnosis a predictor of neurodevelopmental outcome after cardiac surgery in infancy? J Thorac Cardiovasc Surg 2010; 140: 1230-1237.

5. Tabbutt S, Gaynor JW, Newburger JW. Neurodevelopmental outcomes after congenital heart surgery and strategies for improvement. Curr Opin Cardiol 2012; 27: 82-91.

6. Mussatto KA, Hoffmann RG, Hoffman GM, et al. Risk and prevalence of developmental delay in young children with congenital heart disease. Pediatrics 2014; 133: e570-e577. 
7. Ohye RG, Sleeper LA, Mahony L, et al. Comparison of shunt types in the Norwood procedure for single-ventricle lesions. $\mathrm{N}$ Engl $\mathrm{J}$ Med 2010; 362: 1980-1992.

8. Newburger JW, Sleeper LA, Bellinger DC, et al. Early developmental outcome in children with hypoplastic left heart syndrome and related anomalies: the single ventricle reconstruction trial. Circulation 2012; 125: 2081-2091.

9. Goldberg CS, Lu M, Sleeper LA, et al. Factors associated with neurodevelopment for children with single ventricle lesions. J Pediatr 2014; 165: 490-496e8.

10. Bellinger DC, Wypij D, Rivkin MJ, et al. Adolescents with d-transposition of the great arteries corrected with the arterial switch procedure: neuropsychological assessment and structural brain imaging. Circulation 2011; 124: 1361-1369.

11. Sananes R, Manlhiot C, Kelly E, et al. Neurodevelopmental outcomes after open heart operations before 3 months of age. Ann Thorac Surg 2012; 93: 1577-1583.

12. H.R. 1350 - 108th Congress: Individuals with Disabilities Education Improvement Act of 2004. Retrieved from https://www. govtrack.us/congress/bills/108/hr1350.

13. Anderson LM, Shinn C, Fullilove MT, et al. The effectiveness of early childhood development programs. A systematic review. Am J Prev Med 2003; 24 (Suppl): 32-46.

14. Grant R, Isakson EA. Regional variations in early intervention utilization for children with developmental delay. Matern Child Health J 2013; 17: 1252-1259.

15. McManus B, McCormick MC, Acevedo-Garcia D, Ganz M, Hauser-Cram P. The effect of state early intervention eligibility policy on participation among a cohort of young CSHCN. Pediatrics 2009; 124 (Suppl 4): S368-S374.

16. Squires J, Potter L, Bricker D. Ages and Stages Questionnaire, 2nd edn. Brookes Publishing Company, Baltimore, MD, 1999.

17. Roberts G, Howard K, Spittle AJ, Brown NC, Anderson PJ, Doyle LW. Rates of early intervention services in very preterm children with developmental disabilities at age 2 years. J Paediatr Child Health 2008; 44: 276-280.

18. Feinberg E, Silverstein M, Donahue S, Bliss R. The impact of race on participation in part C early intervention services. J Dev Behav Pediatr 2011; 32: 284-291.

19. Hack M, Breslau N, Aram D, Weissman B, Klein N, Borawski-Clark E. The effect of very low birth weight and social risk on neurocognitive abilities at school age. J Dev Behav Pediatr 1992; 13: 412-420.
20. Flores G, Olson L, Tomany-Korman SC. Racial and ethnic disparities in early childhood health and health care. Pediatrics 2005; 115: e183-e193.

21. Glantz S. Primer of Biostatistics, 3rd edn. McGraw-Hill, Inc., New York, NY, 1992.

22. Marino BS, Lipkin PH, Newburger JW, et al. Neurodevelopmental outcomes in children with congenital heart disease: evaluation and management: a scientific statement from the American Heart Association. Circulation 2012; 126: 1143-1172.

23. Soto CB, Olude O, Hoffmann RG, et al. Implementation of a routine developmental follow-up program for children with congenital heart disease: early results. Congenit Heart Dis 2011; 6: 451-460.

24. Brosig C, Butcher J, Butler S, et al. Monitoring developmental risk and promoting success for children with congenital heart disease: recommendations for cardiac neurodevelopmental follow-up programs. Clin Pract Pediatr Psychol 2014; 2: 153-165.

25. Mussatto KA, Hoffmann RG, Hoffman GM, et al. Risk factors for abnormal developmental trajectories in young children with congenital heart disease. Circulation 2015; 132: 755-761.

26. Brosig C, Mussatto K, Hoffman G, et al. Neurodevelopmental outcomes for children with hypoplastic left heart syndrome at the age of 5 years. Pediatr Cardiol 2013; 34: 1597-1604.

27. Medoff-Cooper B, Irving SY, Hanlon AL, et al. The association among feeding mode, growth, and developmental outcomes in infants with complex congenital heart disease at 6 and 12 months of age. J Pediatr 2016; 169: 154-159.e1.

28. Ricci MF, Alton GY, Ross DB, et al. Gastrostomy tube feeding after neonatal complex cardiac surgery identifies the need for early developmental intervention. J Pediatr 2016; 169: 160-165.e1.

29. Wang CJ, McGlynn EA, Brook RH, et al. Quality-of-care indicators for the neurodevelopmental follow-up of very low birth weight children: results of an expert panel process. Pediatrics 2006; 117: 2080-2092.

30. Hadders-Algra M. General movements: a window for early identification of children at high risk for developmental disorders. J Pediatr 2004; 145 (Suppl): S12-S18.

31. Licht DJ, Shera DM, Clancy RR, et al. Brain maturation is delayed in infants with complex congenital heart defects. J Thorac Cardiovasc Surg 2009; 137: 529-536. 